\title{
RADIOLOGIA FORENSE E SUA ATUAÇÃO: UMA BREVE REVISÃO
}

\author{
Gil Dutra FURTADO'; ${ }^{\text {; Felipe Eduardo da Silva SOBRAL }}$; ; Aleudson dos Santos SILVA ${ }^{\mathbf{3}}$; \\ Ádrya Hybia de Lima QUIRINO ${ }^{4}$; Ana Carolina Assis SAMPAIO 5
}

\begin{abstract}
${ }^{1}$ Graduando em Medicina Veterinária/Centro Universitário Maurício de Nassau, João Pessoa (UNINASSAU); Engenheiro Agrônomo/Universidade Federal da Paraíba (UFPB); Doutor em Psicobiologia/Universidade Federal do Rio Grande do Norte (UFRN); Agrônomo-Sócio da Cooperativa de Agronegócio (COOPAGRO), Brasil. Email: gdfurtado@hotmail.com

${ }^{2}$ Médico Veterinário/Universidade Federal de Campina Grande (UFCG); Mestre em Zootecnia/UFCG; Professor de Medicina Veterinária/UNINASSAU, Brasil. E-mail: felipe_eduardo_med_vet@hotmail.com

${ }^{3}$ Graduando em Medicina Veterinária/UNINASSAU; Pedagogo com habilitação em Supervisão Escolar/Centro Universitário de João Pessoa (UNIPÊ), Brasil. E-mail: aleudson@gmail.com

${ }^{4}$ Graduando em Medicina veterinária/UNINASSAU; Técnica em Enfermagem Veterinária, Brasil. E-mail: adryahybia@gmail.com

${ }^{5}$ Graduanda em Administração/UFPB, Brasil. E-mail: anacassissampaio@gmail.com
\end{abstract}

Resumo. Em conjunto com a Medicina Legal, a Radiologia Forense atua realizando exames radiológicos com fins de auxiliar o poder judiciário na elucidação de crimes. Este trabalho enfocou a atuação do profissional em radiologia nas atividades forenses. Para tanto utilizou-se o método revisão bibliográficas, realizada através de livros, artigos científicos e bancos de dados de bases eletrônicas. Observou-se que a radiologia forense é parceira da Medicina Legal, sendo o radiologista um profissional essencial na formação de uma equipe específica para as atividades forenses, auxilia na recuperação das provas, no diagnóstico de causa sobre morte de vítimas humanas e animais.

Palavras-chave: Exames radiológicos; Medicina Legal; Tecnólogo em Radiologia.

\section{FORENSIC RADIOLOGY AND ITS ACTION: A BRIEF REVIEW}

Abstract. In conjunction with Forensic Medicine, Forensic Radiology performs radiological examinations in order to assist the judiciary in elucidating crimes. This work focused on the professional performance in radiology in forensic activities. For this purpose, the bibliographic revision method was used, carried out through books, scientific articles and databases of electronic databases. It was observed that forensic radiology is a partner of Legal Medicine, the radiologist is an essential professional in the formation of a specific team for forensic activities, it assists in the recovery of the evidence, in the diagnosis of the cause of death of human and animal victims.

Keywords: Radiological examinations; Forensic Medicine; Technologist in Radiology. 


\section{RADIOLOGÍA FORENSE Y SU ACTITUD: UNA BREVE REVISIÓN}

Resumen. En conjunto con la Medicina Legal, la Radiología Forense actúa realizando exámenes radiológicos con fines de auxiliar al poder judicial en la elucidación de crímenes. Este trabajo enfocó la actuación del profesional en radiología en las actividades forenses. Para ello se utilizó el método de revisión bibliográfica, realizada a través de libros, artículos científicos y bases de datos de bases electrónicas. Se observó que la radiología forense es un socio de la Medicina Legal, siendo el radiólogo un profesional esencial en la formación de un equipo específico para las actividades forenses, auxilia en la recuperación de las pruebas, en el diagnóstico de causa sobre muerte de víctimas humanas y animales.

Palabras clave: Exámenes radiológicos; Medicina Legal; Tecnólogo en radiología.

\section{INTRODUÇÃO}

De acordo com o Conselho Nacional de Técnicos em Radiologia, a radiologia forense é uma área pertencente ao radiodiagnóstico. Na prática forense a radiologia é considerada um método prático, rápido e objetivo que auxilia no poder judiciário como prova para elucidar diversos tipos de crimes (CONTER, 2014).

Em 1986, Wilhelm Conrad Röentgen detectou uma bala alojada em uma vítima utilizando a técnica de radiologia, na qual por meio do uso do raio $\mathrm{X}$ observou-se a imagem de um projétil alojado no corpo de um cadáver e este serviu como prova para elucidar tal crime (CARVALHO et al., 2009). A radiologia forense engloba, a exemplo do primeiro caso citado, o trabalho de averiguação, interpretação e ponderação dos exames e procedimentos radiológicos efetuados numa investigação criminal (KAHANA; HISS, 1999).

Segundo Sousa et al. (2017), a Radiologia Forense para desenvolver bem suas atividades e atender aos objetivos da profissão, envolve diversos conhecimentos de cunho técnicos científicos como a física, ciências biologia, a ciências químicas, dentre muitas outras.

A atividades de Radiologia Forense pode ser observada nas áreas de atuação da Antropologia forense, traumatologia, asfixiologia, sexologia, obstetrícia forense, antropologia física forense e dactiloscopia (SOUSA et al., 2017).

Em todas estas ramificações a finalidade é auxiliar na resolução de casos, onde se observam dificuldades no seu esclarecimento, devido a vários fatores como a decomposição (biológica ou não) de corpos, carbonização dos corpos e falta de partes do corpo, bem como contribuir para elucidar a provável causa que levou a morte do ser em questão. 
O presente estudo tem por finalidade, buscar informações referentes a radiologia forense, contribuindo para esclarecer a importância desta atuação do profissional de radiologia na área de investigação forense.

Diante do exposto, este trabalho objetiva trazer esclarecimentos acerca da radiologia forense, sendo o foco específico ressaltar a importância desta profissão na elucidação de crimes.

\section{MATERIAL E MÉTODOS}

A fim de atingir o objetivo deste estudo, optou-se pelo método de revisão bibliográfica. A pesquisa bibliográfica foi feita a partir do levantamento de referências teóricas já analisadas, e publicadas por meios escritos e eletrônicos, como livros, artigos científicos, páginas de web sites. Esta metodologia proporciona ao artigo uma cobertura de uma gama de fenômenos muita mais ampla do que aquela que poderia pesquisar diretamente (GIL, 2008). Neste sentido, tal método apresentou a possibilidade de uma melhor visão sobre o tema estudado, proporcionando conhecimentos prévios sobre o tema pesquisado (FONSECA, 2002, p. 32).

\section{RESULTADOS E DISCUSSÃO}

No Instituto de Medicina Legal (IML), as técnicas da radiologia forense podem ser bem utilizadas no auxílio e na recuperação de provas diversas. As atividades desenvolvidas pelo tecnólogo em radiologia são executadas junto ao médico legista e/ou legista. É preciso ter conhecimentos sobre várias áreas da ciência, como física, biologia e química, entre outras que viabilizarão ao profissional desenvolver um bom trabalho de perícia. São várias as áreas de atuação, como examinar as causas da morte, retratando e reconstituindo a cena da morte, através do exame dos ossos e das lesões, com o auxílio de criminalistas e médicos forenses; investiga as lesões corporais resultantes de traumatismos de ordem física; identifica perturbação oriunda da privação, completa ou incompleta, rápida ou lenta, externa ou interna, do oxigênio; atua em processos de identificação humana, em casos de acidentes em massa, crimes e demais situações onde os corpos se encontram carbonizados, em avançado estado de decomposição ou esqueletizados; entre outras situações (ANDRADE, 2016; SOUSA et al., 2017). Em todas estas, o objetivo sempre será auxiliar na resolução de casos difíceis de identificação, corroborando na elucidação da causa do falecimento (RUTTY; MORGAN, 2013). No Instituto Médico Legal (IML) muitos dos corpos recebidos estão no estado de decomposição é comum ter partes do corpo pendentes e/ou carbonizado, dificultando a 
identificação de importantes informações para esclarecer os motivos que contribuíram para a morte do indivíduo (SILVEIRA, 2015).

Os profissionais que atuam com a Radiologia Forense devem ter conhecimento e capacidade técnica, pensamento crítico e de análise, além de estar familiarizado com o funcionamento do nosso sistema judicial (CARVALHO et al., 2009).

Para se realizar uma identificação humana, vários métodos podem ser utilizados, como imagens de odontologia legal, radiografia comum, radiografia digitalizada e até tomografia computadorizada (SILVEIRA, 2015). Curiosamente estas mesmas técnicas podem ser utilizadas, além de em cadáveres e restos humanos, em seres vivos, com o objetivo de identificar foragidos da justiça, desaparecidos, menores de idade, recusa de identificação, dentre outros (FRANÇA, 2011; RIBEIRO, 2015; SIMAS; CALISTO, 2001).

Hishmat et al. (2014), afirma que métodos como a ressonância magnética e a tomografia computadorizada podem ser utilizadas na área forense. Ainda de acordo com esses autores com a utilização de softwares avançados é possível determinar reconstruções faciais em 3D, o que permite que restos mortais sem identificação possam ser reconhecidos por familiares. Este relata ainda que é possível identificar em corpos carbonizados características referentes ao gênero, além de analisar através da densidade óssea se o indivíduo tratava-se de um jovem ou um idoso, em casos de fratura, se esta ocorreu antes ou depois do óbito, dentre outra (RUTTY e MORGAN, 2013). Dados semelhantes puderam ser relatados por Kahana e Hiss (1999), sendo relatada que a radiologia forense pode ser aplicada ainda na detecção de corpos estranhos, na supervisão de bagagens, na inspeção de entrada e/ou saída de drogas, armas e/ou objetos proibidos, em aeroportos e presídios do país.

De acordo com a Lei n ${ }^{\circ}$ 564/99 de 21 de dezembro de 1999, o Técnico de Radiologia é o profissional responsável pela realização de todos os exames da área da radiologia, utilizando técnicas e normas de proteção e segurança radiológica no manuseamento com radiações ionizantes. $\mathrm{Na}$ área da radiologia forense os técnicos de radiologia devem assegurar que os exames sejam realizados de maneira adequada (KAHANA; HISS, 1999) já que estão realizando um trabalho que exige precisão e responsabilidade.

Desta forma, mesmo com a importância que esta função tem, o que pode ser observado de acordo com Rutty e Morgan (2013), é que apesar das inúmeras vantagens da implementação da radiologia forense está ainda é considerada uma área em desenvolvimento haja vista que ainda não existem padrões internacionais para potenciais radiologistas ou patologistas que na área forense. Observa-se, portanto uma carência de investimentos econômicos na formação dos profissionais desta área. 
Para um tecnólogo em radiologia capacitado a desenvolver estas atividades, a exemplo das atividades relacionadas aos serviços de radiologia e diagnóstico por imagens, é considerado (o tecnólogo) um recurso de alto potencial e por isso o investimento realizado pelas faculdades, universidades e institutos federais são indispensáveis para se obter um profissional que traga muitos benefícios para a sociedade em geral.

Para Vicente-Ramírez, Fernández e Caravaca (2017) o técnico ou tecnólogo médico em radiologia tem o dever de minimizar a dose para os pacientes e a população como um todo; portanto, realizar este tipo de exploração entra em conflito com os princípios da proteção radiológica e de seu código de ética

Cavallari (2017) também observa que a virtópsia vem se mostrando um método superior e complementar em relação aos métodos tradicionais de autópsia e possui muitas vantagens, no entanto, ainda são necessários estudos para substituir por completo a autópsia convencional por exames de imagens (CAVALLARI et al., 2017).

Em uma ampliação da atividade da Radiologia Forense, observa-se que na medicina veterina este profissional também tem seu espaço de atuação. Em medicina veterinária, a técnica de radiologia forense tem ajudado a perícia principalmente em crimes relacionados à morte inesperada de animais, bem-estar, abuso e violações da lei de conservação, a caça e a maus-tratos (COOPER; COOPER, 2008; CORRADI; LUPPI; PITTIONI, 2014). De acordo com alguns especialistas, o objetivo para na medicina veterinária envolve "fornecer as informações necessárias para uma posterior decisão judicial acerca de um determinado caso, assegurando que todas as provas recolhidas sejam válidas".

$\mathrm{Na}$ medicina veterinária, as principais aplicações da radiologia forense se destacam nas técnicas de imagem de radiografia (Figs. 1 e 2), radiografia pós-mortem, ultrassonografia, tomografia computadorizada, angiotomografia e a ressonância magnética, além de realçar a importância dessas práticas no auxílio da necropsia convencional, como também, no avanço do diagnóstico por imagem na ciência forense. 


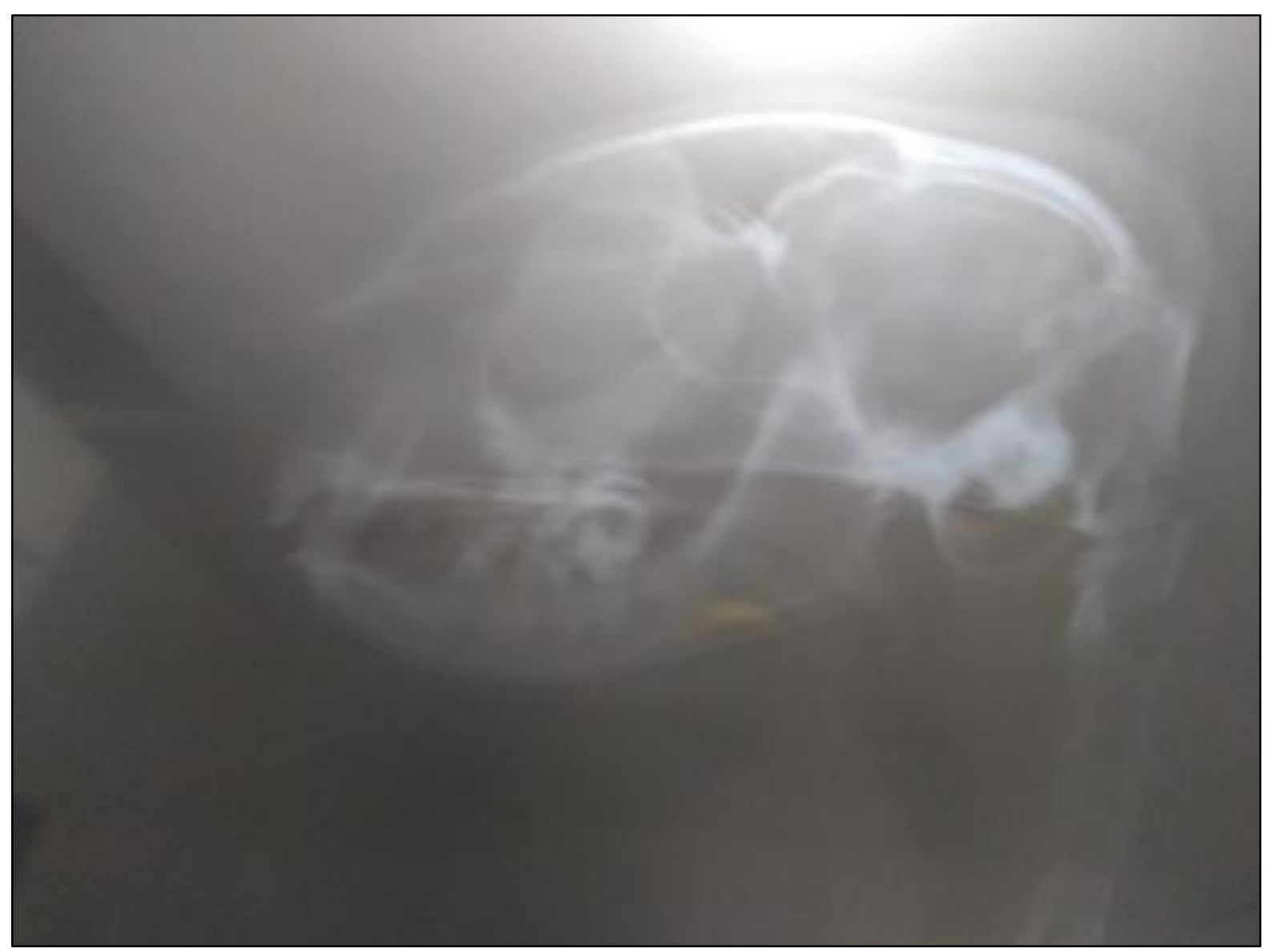

Figura 1. Radiografia do crânio de um felino. Fonte: cedida pela Uniserv clínica veterinária. 


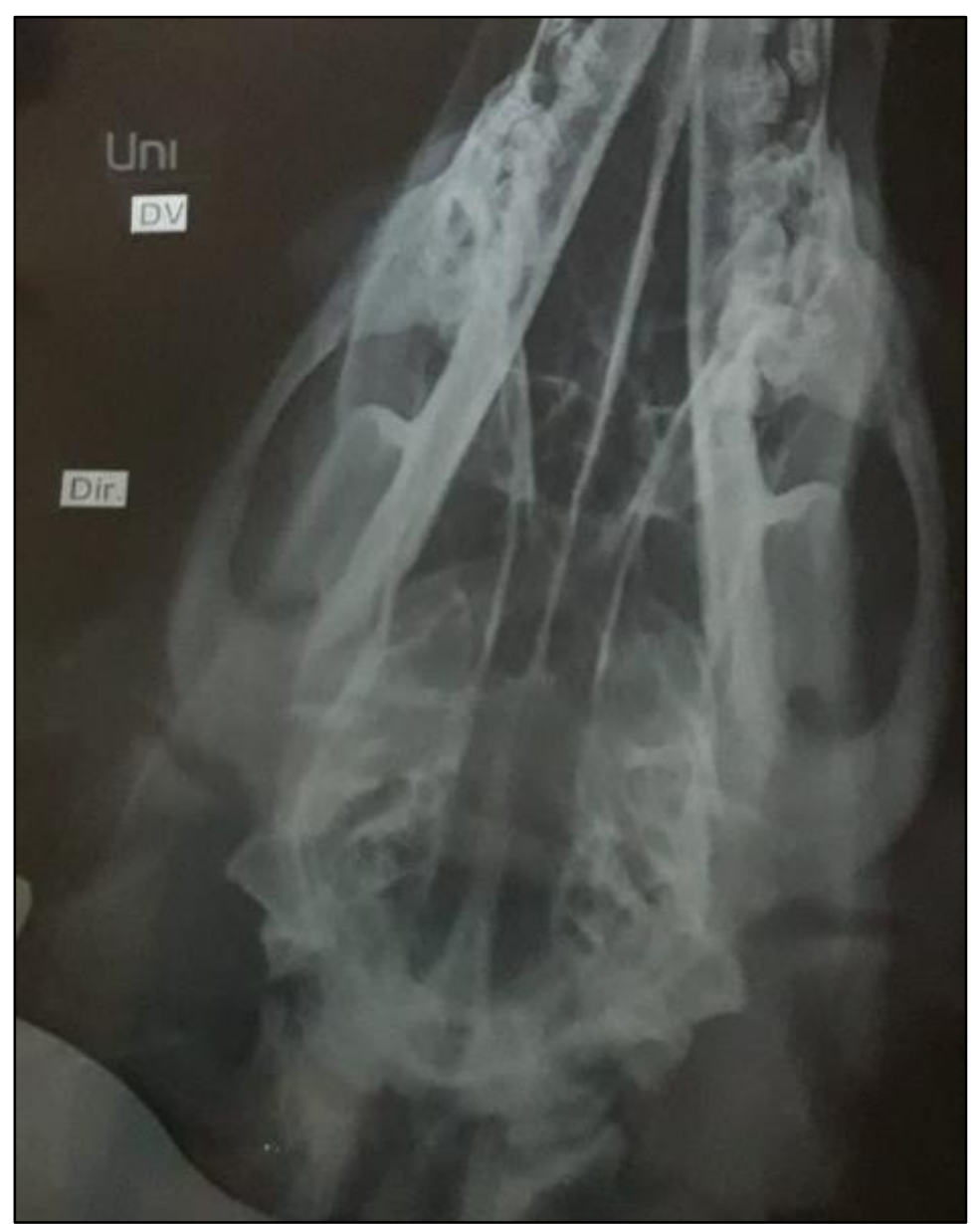

Figura 2. Radiografia da mandíbula de um canídeo. Fonte: os autores.

\section{CONCLUSÕES}

Diante do exposto, conclui-se que a radiologia forense é de grande importância para o diagnóstico de mortes diversas na medicina legal, auxiliando na identificação da causa morte, além de atuar em outras áreas, como nos presídios e aeroportos na supervisão das bagagens, identificando drogas, armas, objetos proibidos, na Medicina Veterinária, dentre outros. Ressalta-se ainda a grande valia do trabalho do tecnólogo em radiologia, que juntamente com o médico legista, podem atuar de forma a obter um diagnóstico mais preciso e mais rápido.

\section{AGRADECIMENTOS}

Os autores agradecem a colaboração dos alunos Djonny MICHELL; Lucas PEREIRA; Maria ANGÉLICA; Rogério Ferreira DIAS e Viviany CHRISTINE; todos Graduandos do curso de tecnologia em radiologia das Faculdades de Enfermagem e de Medicina Nova Esperança FACENE/FAMENE, que contribuíram muito para a realização deste artigo. Agradecemos especialmente à empresa "Uniserv clínica veterinária" pela contribuição científica concedida. 


\section{REFERÊNCIAS}

ANDRADE, S.A.F.; A atuação do técnico e do tecnólogo em radiologia na área forense. UNILUS Ensino e Pesquisa, Santos, v. 13, n. 30, p. 26-31, 2016.

CARVALHO, S.P.M.; SILVA, R.H.A; LOPES, Jr.C.; SALES-PERES, A. A utilização de imagens na identificação humana em odontologia legal. Radiologia Brasileira, São Paulo, v. 42, n. 2, p. 125-130, 2009.

CAVALLARI, E.F.; PICKA, M.C.M.; PICKA, M.C.M. O uso da tomografia computadorizada e da ressonância magnética na virtópsia. Tekhne e Logos, Botucatu, v. 8, n. $1,2017$.

CONTER (Conselho Nacional de Técnicos em Radiologia). Radiologia Forense: História, aplicações e mercado de trabalho. Disponível em: http://www.conter.gov.br/site/noticia/profissao-rx. Acesso em: 17 nov. 2018.

COOPER, J.E.; COOPER, M.E. Forensic veterinary medicine: a rapidly evolving discipline.

Forensic Science, Medicine and Pathology, v. 4, n. 2, p. 75-82, 2008.

CORRADI, A.; LUPPI, A.; PITTIONI, E. Medicina forense veterinaria e lesioni da arma da fuoco: caratteri e diagnosi. 2014. Disponível em: https://www.researchgate.net/publication/237658145. Acesso em: 2 dez. 2018.

FRANÇA G.V. Medicina legal. 9 ed. Rio de Janeiro: Guanabara Koogan, 2011.

FONSECA, J.J.S. Metodologia da pesquisa científica. Fortaleza: Universidade Estadual do Ceará, 2002. Apostila.

GIL, A.C. Métodos e Técnicas de Pesquisa Social. 6.ed. São Paulo: Atlas, 2008.

GRABHERR, S.; DJONOV, V.; YEN, K.; THALI, M.J.; DIRNHOFER, R. Postmortem angiography: Review of former and current methods. American Journal of Roentgenology, v. 188, p. 832-838, 2007. 
HISHMAT, A.M.; MICHIUE, T.; SOGAWA, N.; ORITANI, S.; ISHIKAWA, T.; HASHEM, M.A. MOEDA, H. Efficacy of automated three-dimensional image reconstruction of the femur from postmortem computed tomography data in morphometry for victim identification. Legal Medicine, v. 16, p.114-117, 2014.

KAHANA, T.; HISS, J. Forensic radiology. The British Journal of Radiology, v. 72, p. 12933, 1999.

POMPEO, D.A.; ROSSI, L.A.; GALVAO, C.M. Revisão integrativa: etapa inicial do processo de validação de diagnóstico de enfermagem. Acta Paulista de Enfermagem, v. 22, n. 4, p. 434-438, 2009.

RIBEIRO, A.B. Relampejos do passado: inscrição da morte no espaço público através da exumação de corpos de desaparecidos políticos da ditadura militar brasileira. $112 \mathrm{f}$. Dissertação (Mestrado em Antropologia Social)-Faculdade de filosofia, Letras e Ciências Humanas, Universidade de São Paulo, São Paulo, 2015.

RUTTy, G.N. MORGAN, B., 2013. Virtual autopsy. Forensic Science, Medicine, and Pathology, v. 9, p. 432, 2013.

SILVEIRA, P.R. Fundamentos da Medicina Legal. Lumen Juris: Rio de Janeiro, 2015

SIMAS, A. CALISTO, F. Lofoscopia. Loures: Instituto Superior de Polícia Judiciária e Ciências Criminais, 2001.

SOUSA, B.L.M.; SEREJO, F.S.; DUARTE， H.; CANTANHEDO, J.J.B.; SILVA, M.; ROCHA, W.S.; SOARES, W.D.B. Radiologia Forense na Área Criminal. Revista Científica Multidisciplinar Núcleo do Conhecimento. v. 13, p. 455-462, 2017. Ano 02, ed. 1.

VETTORATO, M.C.; FOGAÇA, J.L.; TREVISAN, A.C.; RAHAL, S.C.; FERNANDES, M.A.R. Principais aplicações daradiologia forense em medicina veterinária $6^{\mathbf{a}}$ Jornada Científica e Tecnológica da FATEC de Botucatu 23 a 27 de Outubro de 2017, Botucatu - São Paulo, Brasil. 
VICENTE-RAMÍREZ, R.M.; FERNÁNDEZ, J.L.B.; CARAVACA, G.R. Radiología médicolegal. Un dilema ético para el técnico en radiología. Acta Bioethica, Santiago, v. 23, n. 2, p. 245-251, 2017. 